\title{
Three-Dimensional Ultrasound versus Hysteroscopy in Uterine Cavity Assessment after Failed Intracytoplasmic Sperm Injection: a study for accuracy of a diagnostic test
}

\author{
Faten Mosbah Ibrahim Al-Zinaty ${ }^{1}$, Mohammed Sayed Ali ${ }^{2}$, Mohammed Mahmoud Al \\ Sherbeeny ${ }^{2}$, Tarek Aly Raafat \\ ${ }^{1}$ Obstetrics and Gynecology Clinics, Al-Tayseer Clinics, ${ }^{2}$ Department of Obstetrics and Gynecology, Faculty of \\ Medicine, Ain-Shams University \\ Corresponding author: Faten Mosbah Ibrahim Al-Zinaty, Email: fatenmosbah@ hotmail.co.uk, Phone: +201222117730.
}

\begin{abstract}
Background: Implantation failure after intracytoplasmic sperm injection (ICSI) is related to either maternal factors or embryonic causes. The maternal factors include uterine anatomic abnormalities either congenital or acquired. Three-dimensional transvaginal ultrasound allows detailed evaluation of pelvic organs. Office hysteroscopy is the gold standard tool for visualization of intracavitary lesions and the cervical canal in an office-based environment.

Aim of The Work: It was to compare between three-dimensional ultrasound (3D USS) and hysteroscopy for evaluation of the intrauterine cavity after trial of ICSI and to study the accuracy of diagnostic test in a tertiary care facility.

Patients and Methods: It was a prospective; blinded; controlled clinical trial comparative study. Both hysteroscopy and 3D USS procedures were done for uterine cavity assessment after failed attempt of ICSI treatment for fifty two asymptomatic infertile women.

Results: Seventeen cases of our studied patients were without intracavitary or cervical lesions. From all studied cases, lesions $(n=66)$ were seen either by hysteroscopy or by 3D USS where lesions $(n=24)$ were seen by hysteroscopy and seen by ultrasound. Lesions $(n=34)$ were seen by hysteroscopy. Lesions $(n=36)$ were seen by ultrasound. Measure of agreement of Kappa was 0.273. Overall sensitivity of 3D USS was $70.59 \%$ and specificity $62.50 \%$ with positive predictive value (PPV) $66.67 \%$ and negative predictive value (NPV) $66.67 \%$, accuracy was $66.67 \%$, positive likehood ratio (LR+ve) $1.88 \%$ and negative likehood (LR-ve) $0.47 \%$. The pvalue was 0.026 , which is statistically significant.

Conclusion: It is important to investigate the uterine cavity in infertile women with history of unsuccessful ICSI. 3D USS has a good sensitivity and specificity for diagnosis of uterine cavity abnormalities and it could be used before hysteroscopy as a first line of investigation of intrauterine lesions.
\end{abstract}

Key Words: Intracytoplasmic Sperm Injection (ICSI), Repeated implantation failure (RIF), Hysteroscopy, Three-Dimensional Ultrasound (3D USS), Gold Standard, Sensitivity, Specificity.

\section{INTRODUCTION}

Intracytoplasmic sperm injection (ICSI) is an assisted reproductive technology (ART) used to treat sperm-related infertility problems ${ }^{(1)}$.

ICSI was introduced in 1992 to improve fertilization in couples with or without detectable abnormalities of semen parameters ${ }^{(2)}$. ICSI has become the premier treatment modality for severe male factor infertility, yielding excellent pregnancy and implantation rates ${ }^{(3)}$.

However, even after ICSI, complete failure of fertilization occurs in 1-3\% of cycles. Most cases occur due to low number of mature oocytes, failure of oocyte activation or non-availability of appropriate spermatozoa for injection ${ }^{(4)}$.

The probable causes of repeated in vitro fertilization (IVF) failures are classified as: reduced endometrial receptivity, embryonic defects or multifactorial causes. Intrauterine and endometrial integrity abnormalities such as thin endometrium, altered expression of adhesive molecules and immunological factors like Anti Sperm (ASA), Anticardiolipin (aCL), Lupus anticoagulant (LA), Anti-Phosphatidylserine (aPS), Anti-Phosphatidylethanolamine (aPE), and Antinuclear antibody (ANA), Anti-DNA, Anti-Zona and Anti-ovarian (AOA) antibodies, Thrombophilia, decrease expression of endometrial integrins, increase of natural killer cells activities and imbalance of cytokine networks (balance between IL-12 and IL-18), may decrease endometrial receptivity, whereas chromosomal and genetic abnormalities of the male sperm or female ovarian defects, embryonic aneuploidia or zona hardening are embryonic reasons for the failure of implantation. Among the various etiologies that where, endometrial regularity due to anatomical malformations of the uterus, either congenital (septate uterus and bicomuate uterus) or acquired (uterine fibroids, especially submucous type, endometrial polyps, intrauterine adhesions and hydrosalpinx), play an important role in infertility and success of IVF programs ${ }^{(5)}$. 
Recurrent implantation failure (RIF) is a challenging and extremely disappointing problem faced by the clinicians and the couples alike. The

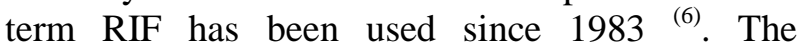
implantation rate per embryo transferred usually does not exceed $30 \%$, although higher rates with the use of blastocysts have been reported, depending on female age ${ }^{(7)}$.

In women with unexplained RIF, although they have good hormonal response, good-quality embryos, satisfactory endometrial development and no identifiable pathology, suboptimal endometrial receptivity is considered a key factor in inhibiting embryo implantation. During the implantation window, there is a cross-talk between the embryo and the endometrium to allow attachment, adhesion and invasion of the embryo ${ }^{(8)}$.

It is widely accepted that a complete infertility workup should include an evaluation of the uterine cavity. Uterine abnormalities, congenital or acquired, are implicated as one of the causes of infertility ${ }^{(9)}$.

The prevalence of such intrauterine abnormalities, diagnosed by hysteroscopy prior to IVF, has been described to be between 20 and $45 \%{ }^{(10)}$.

Conventional two-dimensional ultrasound (2D-USS) is widely used in gynecological practice in the work up of infertility and it may be considered as an essential imaging technique for diagnosing uterine and adnexal pathology (11). Three-dimensional transvaginal ultrasound (3D TVS) provides a unique diagnostic tool for noninvasive studies of the uterine morphology and diagnosis of congenital uterine anomalies ${ }^{(12)}$.

The three-dimensional volume can be manipulated in several ways. Probably the most used and useful display is multiplanar display, which simultaneously shows three orthogonal planes (axial, longitudinal, and coronal) allowing navigation through these three planes. The coronal plane is almost impossible to obtain in conventional vaginal ultrasound and difficult to obtain in abdominal ultrasound but easy to reconstruct using 3D USS ${ }^{(11)}$.

Hysteroscopy is known as the gold standard procedure for uterine cavity assessment and visualization of intracavitary lesions and the cervical canal ${ }^{(13)}$. Hysteroscopy, however, is a diagnostic procedure that makes available direct demarcation of endometrial submucosal, intrauterine cavity deformity and even the cervical canal ${ }^{(14)}$. It enables diagnosis and treatment of intrauterine pathology in the same outpatient setting. Hysteroscopy is quick, safe and well-tolerated procedure ${ }^{(13)}$. Therefore, it has become an excellent tool for the diagnostic and therapeutic infertility work-up. It has been frequently advised to perform hysteroscopy as a routine procedure prior to IVF/ICSI treatment ${ }^{(10)}$.

\section{AIM OF THE WORK}

It is a study to the evaluate the validity, sensitivity, specificity, positive and negative predictive values of a diagnostic test, to compare between 3D USS and hysteroscopy which is the gold standard tool in the evaluation of the intrauterine lesions after failed ICSI in a tertiary care facility.

\section{PATIENTS AND METHODS}

\section{Study type}

It is prospective; blinded; controlled clinical trial against a gold standard test.

\section{Study population}

Fifty two asymptomatic infertile women with history of failed ICSI treatments were included in this study. The included women in this study were with either primary or secondary infertility, aged 21-46 years with normal baseline hormonal profile (Day 2 FSH, LH, E2, TSH, and PRL). These women were with no detectable pelvic pathology on TVS which done within the previous six months and had history of previous failed ICSI cycle within previous 12 months (one/or more). On the other hand, the exclusion criteria were women with history of pelvic inflammatory disease, chronic pelvic pain, pelvic surgery, severe dysmenorrhea, dyspareunia or polycystic ovarian syndrome (PCOS).

\section{Sample size calculations}

In a test for agreement between two techniques using the Kappa statistic, a sample size of at least 50 subjects achieves $95 \%$ power to detect a true Kappa value of 0.80 in a test of H0: Kappa $=0.40$ vs. H1: Kappa $<>0.40$ when there are two categories with frequencies equal to 0.50 , and 0.50 . This power calculation is based on a significance level of $0.05^{(15 ; 16)}$.

\section{Sample method}

Non-random sampling (convenience).

\section{Ethics statement}

The study was approved by the local Ethics Committee. Informed consent was taken from 
participants includes information about risks and benefits out of the research, and also that participating women's right to withdraw from the study without being adversely impacted.

This study has been conducted at Early Cancer Detection Unit and Ultrasound Special Care Unit for the Foetus at Ain Shams University Maternity Hospital, Egypt. The study started on January 2015 till November 2016 i.e. about two years.

\section{Study methods}

For all patients a complete history was taken and a physical examination was done. For male partner, evaluation was also done and included a medical history and a clinical examination. Semen analysis included two separate samples with at least a 2-week interval and an interpretation according to World Health Organization (WHO) criteria.

Hysteroscopy was done as routine procedure for uterine cavity assessment after failed attempt of IVF/ICSI treatment and the patients had an ultrasound assessment of uterine cavity with a 3D USS as well.

Both hysteroscopy \& 3D USS procedures were scheduled at post menstrual period in the earlymid follicular phase of a cycle of the same menstrual cycle, 1-3 months before the next ICSI treatment. All patients received non- steroidal analgesic $30 \mathrm{~min}$ before the hysteroscopic procedure and prophylactic antibiotics (200 mg of doxycycline before \& $100 \mathrm{mg}$ twice daily for five days after).

Hysteroscopy: office hysteroscopies was carried out in a standardized manner, using a $2.9 \mathrm{~mm}$ outer-diameter continuous flow Bettocchi hysteroscope with $30^{\circ}$ direction of view (Karl Storz Endoscopy, America Inc. USA or GmbH \& co. KG Germany). Normal sterile, isotonic saline solution was used for distension of the uterine cavity. The uterine cavity was assessed on its shape (normal, arcuate or septate) and the presence or absence of abnormalities (endometrial polyps, myomas, adhesions and septa). The findings during hysteroscopy were recorded on digital optical disc (DVD) and reviewed by senior gynecologists. Any uterine abnormalities diagnosed in the studied cases were treated using operative hysteroscopy and specimens obtained were sent for histopathological examination. The hysteroscopy was done by the most experienced supervisor.

Three-dimensional ultrasound examinations were performed using a Voluson Pro and three Voluson E6 BT12 Expert (GE Medical Systems, Zipf, Austria) ultrasound machines, equipped with endocavitary probe RIC5-9-D-4D. In all cases, we obtain transvaginally between one and three static volumes of the uterus, with a quality ranging from medium to maximum.

Initially we visualized the uterus on 2D USS in a strict mid-sagittal view, adjusting the capture window to obtain the optimal 3D volume. The volume was then obtained using a sweep angle of 90 from one side of the uterus to the other, bisecting the capture plane. The uterine cavity was evaluated with attention to its contour, dimensions, regularity, and thickness of the endometrium and presence of endometrial polyps or fibroids in three planes. 3D USS was done by the same senior sonographer.

\section{Blinding}

The hysteroscopist and the sonographer were blinded about the results of each other.

\section{Statistical Analysis}

Data were collected, tabulated and then statistically analyzed using Statistical Package for Social Sciences (SPSS). Numerical variables were presented as mean $\pm \mathrm{SD}$, while categorical variables were presented as number and percentage. Student $(t)$ test was used for comparison between groups as regard numerical variables. A difference with $p$-value $<0.05$ was considered statistically significant, otherwise it is insignificant. Sensitivity was the proportional detection of individuals with the disease of interest in the population. Specificity is the proportional detection of individuals without the disease of interest in the population. Positive predictive value (PPV) is the proportion of all individuals with positive tests, who have the disease. Negative predictive value (NPV) is the proportion of all individuals with negative tests who are nondiseased. The positive likelihood ratio $(\mathrm{LR}+)=$ sensitivity/ (1- specificity). The sensitivity, specificity, accuracy and the predictive values of the hysteroscopy \&3D USS were calculated using technical notes.

\section{RESULTS}

In our study, the included women $(n=52)$ had a history of previous failed IVF/ICSI, $n=33$ $(63.5 \%)$ with one failed ICSI, $n=14$ (26.9\%) with two failed ICSI, $n=3(5.8 \%)$ with three failed ICSI and $n=2(3.9 \%)$ with four failed ICSI.

Failed ICSI was due to failure of implantation after successful fertilization. Male factor was normal at $n=47(90.4 \%)$ and abnormal at $n=5$ (9.6\%). The initial characteristics of included women 
were at age range from $21-46$ years with mean \pm SD $32.83 \pm 5.43$ with body mass index (BMI) range from $21.1-34.5 \mathrm{~kg} / \mathrm{m}^{2}$ with mean \pm SD $27.1 \pm 2.98$ and duration of marriage range between $1.5-30$ years with mean \pm SD8.7 \pm 5.01

The type of infertility was either primary infertility $n=34(65.4 \%)$, or secondary infertility $n=$ 18 (34.6\%). Menses were regular at $n=47(90.4 \%)$ and irregular at $n=5(9.6 \%)$. Their hormonal profiles were more or less within normal limits.

The selected women within the inclusion criteria were subjected to investigation by hysteroscopy and three-dimensional ultrasound, and both were done at the post menstrual period i.e. at the follicular phase of menstrual cycle for the women with regular cycles and at any time for the women with irregular cycles.

Seventeen cases $(n=17)$ of our studied patients were without intracavitary or cervical lesions, as it is well-known that most of patients history of failed ICSI may be without intracavitary lesion when investigated either by hysteroscopy or ultrasound. From all studied cases $(n=52),(n=$ 66) lesions were shown either by hysteroscopy or by three-dimensional ultrasound where $(n=24)$ lesions were seen by hysteroscopy and seen by ultrasound, $(n=20)$ lesions were not seen by hysteroscopy and not seen by ultrasound. $(N=12)$ lesions were not seen by hysteroscopy but seen by ultrasound, and $(n=10)$ lesions were seen by hysteroscopy but not seen by ultrasound (Table 1 ).

Table (1): Agreement table.

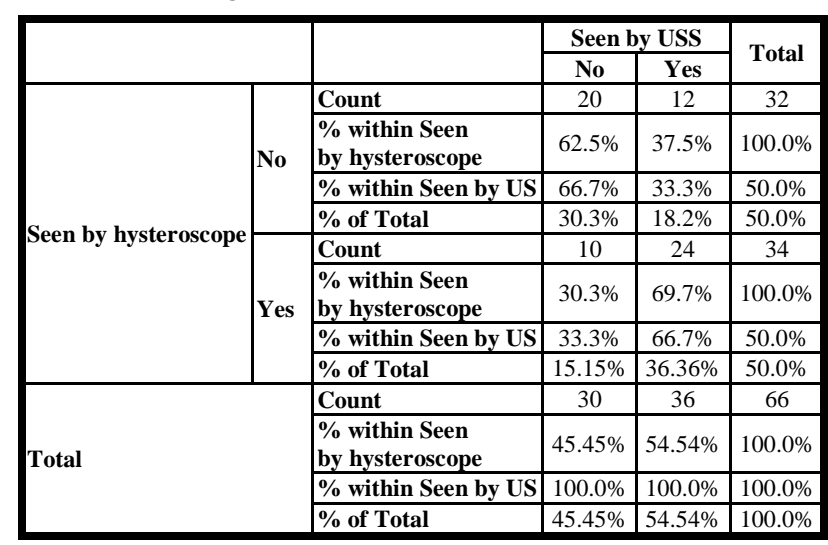

\section{Hysteroscopic findings of included women:}

In our study, $(n=66)$ lesions were shown either by hysteroscopy or by three-dimensional ultrasound. Uterine position was anteverted uterus (AVF) in all cases. Hysteroscopy showed $(n=1)$ polyp at ectocervix, $n=3(4.5 \%)$ endocervical polyps and $n=2(3.0 \%)$ adhesions at endocervical canal.

For the uterine cavity, hysteroscopy showed 26 intracavitary lesions in 14 cases $(25 \%)$. Intrauterine polyps $n=9(13.6 \%)$, adhesions $n=5$ $(7.6 \%)$ of them $(n=2)$ were filmy adhesions and $n$ $=3$ of them were dense adhesions (synaechia), septums $n=9(13.6 \%)$ of them $n=3(4.5 \%)$ were septate vs bicornuate, $n=5(7.6 \%)$ were septate and $n=1(1.5 \%)$ was subarcuate. Submucous myoma $n=2(3.0 \%)$, compression of the uterine cavity $n=2(3.0 \%)$, and endometrial hyperplasia $n$ $=1(1.5 \%)$. Endometrium was normal at $n=4$ $(7.7 \%)$ cases, thin at $n=33(63.5 \%)$ cases, and thick at $n=15$ (28.8\%).

\section{Sonograghic findings of included women:}

Uterine position: AVF $n=48$ (92.3\%), retroverted uterus (RVF) $n=4(7.7 \%)$, cervix: NAD $n=51(98.1 \%)$, and double cervix $n=1$ (1.9\%). 3D USS showed $n=1(1.5 \%)$ endocervical polyp and $(n=25)$ lesions inside the uterine cavity. Intrauterine polyps $n=9(13.6 \%)$, size of polyps range between minimum $2 \mathrm{~mm}$ to maximum 18 $\mathrm{mm}$ with mean of \pm 8.06 . Adhesions $n=5(7.6 \%)$ where $(n=2)$ of them were dense adhesions (synaechia), and one of them $(n=1)$ appeared as narrow irregular cavity. Septum $n=8(12.12 \%)$ of them $(n=1)$ was uterine duplex and $(n=1)$ was bicornuate uterus, myomas were $n=12(18.18 \%)$ of them submucous myoma $n=2(3.0 \%)$, compression of cavity by intramural myoma $n=10$ $(15.15 \%)$, and endometrial hyperplasia $n=1$ (1.5\%). Endometrium: regular $n=46$ (78.8\%), irregular $n=6(11.5 \%)$, myometrium consistency was homogenous at $n=39(75 \%)$ and heterogeneous at $n=13(25.0 \%)$.

Measure of agreement of Kappa (Table 2), the value was 0.273 for $(n=66)$ cases, Asymptotic Standard Error (a) 0.118, where (a) means not assuming the null hypothesis, approx. $\mathrm{T}$ (b) 2.232, where (b) means using the asymptotic standard error assuming the null hypothesis.

Table 2: Symmetric measures.

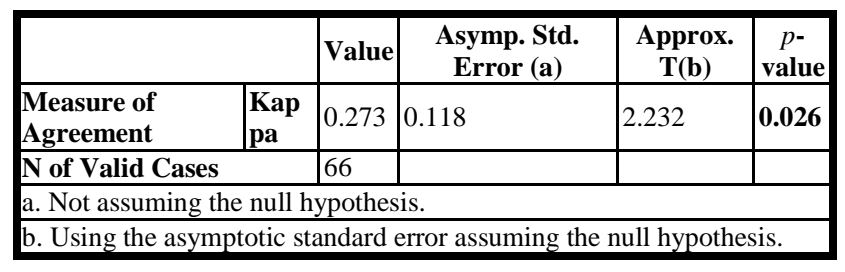


Overall sensitivity was $70.59 \%$ and specificity $62.50 \%$ with PPV $66.67 \%$ and negative predictive value (NPV) 66.67\%, accuracy was $66.67 \%$, (LR+ve) $1.88 \%$ and (LR-ve) $0.47 \%$. The $p$-value was 0.026 so it is significant, as the $p$ values less than 0.05 is considered statistically significant (Table 3, Diagram1).

Table (3): Measurement of accuracy.

\begin{tabular}{|c|c|c|c|c|c|c|c|c|c|c|c|}
\hline Item & TP & FN & TN & FP & Sensitivity & Specificity & $\begin{array}{c}(+) \\
\text { ve PV }\end{array}$ & $\begin{array}{c}(-) \\
\text { ve PV }\end{array}$ & Accuracy & LR+ & LR- \\
\hline 3D USS & 24 & 10 & 20 & 12 & 70.59 & 62.50 & 66.67 & 66.67 & 66.67 & 1.88 & 0.47 \\
\hline
\end{tabular}

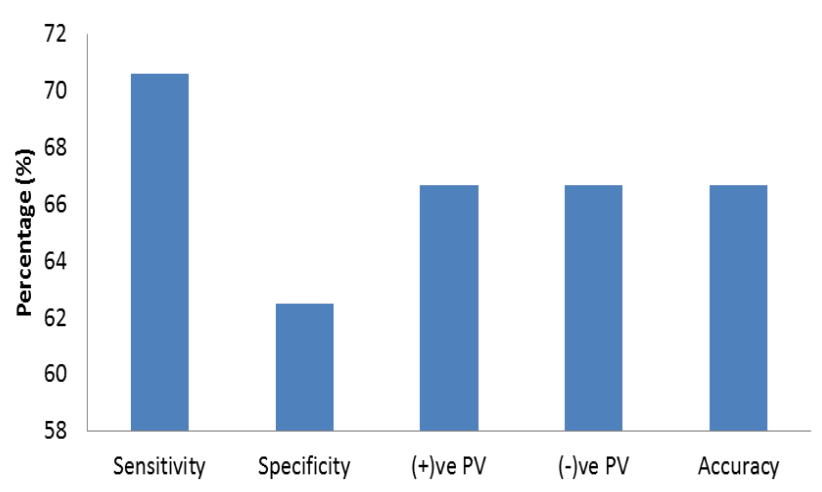

Figure (1): Bar-Chart showing accuracy of 3D TVS in contrast to hysteroscopy in included women.

\section{DISCUSSION}

ICSI is a useful technique for couples that have low or absent fertilization during IVF, or where the sperm quality and count is too low for normal IVF to be successful ${ }^{(17)}$. Failed ICSI may be due to failure in fertilization or may be due to failure in implantation. RIF is determined when embryos of good quality fail to implant following several IVF treatment cycles. Implantation failure is related to either maternal factors or embryonic causes. Maternal factors include uterine anatomic abnormalities, thrombophilia, non-receptive endometrium and immunological factors ${ }^{(18)}$.

3D TVS allows detailed evaluation of pelvic organs by collecting a series of sequential ultrasound images and converting them into an ultrasound volume ${ }^{(19)}$. The three-dimensional volume can be manipulated in several ways. Probably the most used and useful display is multiplanar display, which simultaneously shows three orthogonal planes (axial, longitudinal, and coronal) allowing navigation through these three planes. The coronal plane is almost impossible to obtain in conventional vaginal ultrasound and difficult to obtain in abdominal ultrasound but easy to reconstruct using 3D USS ${ }^{(11)}$. Hysteroscopy is known as the gold standard procedure for uterine cavity assessment and visualization of intracavitary lesions and the cervical canal ${ }^{(13)}$.It is a very useful technique for diagnosis and treatment of uterine pathology in an office-based environment ${ }^{(20)}$.

The aim of the work was to study the accuracy of the (3D USS) against the gold standard tool (hysteroscopy) for detection of intrauterine lesion in infertile women with history of failed ICSI in a tertiary care facility.

In our study, the numbers of endometrial polyps that seen by hysteroscopy are $(n=11)$ polyps, two of them are submucous fibroid polyps with broad base and we considered them as submucous myomas, so we were left with nine mucous endometrial polyps seen by hysteroscopy. On the other side, the number of endometrial polyps seen by 3D USS were $(n=9),(n=4)$ of them were not seen by hysteroscopy so $(n=5)$ polyps were shared and seen by the two tools. However, two polyps which were seen as mucous broad based polyps by hysteroscopy seen as thickened endometrium about $12 \mathrm{~mm}$ in one and 10 $\mathrm{mm}$ in another by 3D USS.

The two submucous myomas seen by hysteroscopy as fibrous broad based polyps were seen by 3D TVS as two intramual myomas encroaching at uterine cavity as about $3 / 4$ of myoma protruded inside the uterine cavity.

For the intrauterine adhesions $(n=2)$ were just filmy adhesions, $(n=1)$ appeared as narrow irregular cavity and $(n=2)$ were dense adhesion which are named synaechia grade I and II. The compression of cavity by intramural myoma seen by hysteroscopy $(n=2)$ while $(n=11)$ seen by 3D USS.

For the septum $(n=8)$ seen by 3D USS, where $(n=6)$ of them were intracavitary septums without indentation of the fundus. From the three septums which were seen as septate vs bicornuate by hysteroscopy, $(n=1)$ was bicornuate uterus with marked indentation of the fundus as confirmed by $3 \mathrm{D}$ USS and $n=1$ was uterine duplex with two cervices as shown by 3D USS as well. Arcuate uterus seen by hysteroscopy was not seen as so by 3D USS and cavity was triangular by coronal view.

We cannot rely completely on hysteroscopy for diagnosing uterine congenital abnormalities especially septate or bicornuate uterus, 3D USS shows if there is indentation of the 
fundus of uterus and will differentiate very clearly between septate uterus with only fibrous septum and bicornuate uterus with muscular septum and for how far we can operate through it.

By 3D TVS we have panoramic view about the myometrium and adnexae. The coronal view allows for examination of the endometrium and the adjacent myometrium and we can see the intramural fibroids and how far they compress the uterine cavity and this will not be achieved by hysteroscopy. Office hysteroscopy has a good sensitivity for diagnosis of endometrial polyps, submucous myomas and intrauterine synechiae.

Overall sensitivity of 3D TVS was $70.59 \%$ and specifity $62.50 \%$ with $p$-value $=0.026$, which is significant. So we can assume the Null hypothesis or Alternative hypothesis which tries to prove that the $3 \mathrm{D}$ TVS is equivalent to hysteroscopy in the diagnosis of intrauterine lesions. If 3D TVS is equivalent to hysteroscopy and has the same sensitivity and specificity, we can use it in diagnosis instead of hysteroscopy as 3D TVS is not invasive tool. As it is not, then hysteroscopy is superior and more accurate than 3D TVS so we can still consider hysteroscopy as the gold standard procedure for uterine cavity assessment.

We agreed with Arefi et al. ${ }^{(5)}$ and other studies which stated that the hysteroscopy has a higher sensitivity and specifity compared to other diagnostic tools (saline infusion hysterosonography 'SIHS', TVS, and 3D USS) and stated that hysteroscopy is the gold standard for the investigation of uterine cavity. It is a safe test for the direct and accurate diagnosis of intrauterine abnormalities ${ }^{(5 ; 21 ; 22)}$.

Our study agreed also with Ebrashy et al. (23) who stated that 3D USS is of great value than hysteroscopy in delineating with certain the exact position of the submucous myomas or endometrial polyps in relation to the cavity. In another study which was done by Karasu and Metwally (24) stated that 3D USS has high sensitivity in the diagnosis of septate uterus and has a low sensitivity $(52 \%)$ in the diagnosis of intrauterine adhesions and we agreed with it. Sylvestre $\boldsymbol{e t}$ al. ${ }^{(25)}$ study clearly demonstrated how simple contrast media potentially can increase the specificity of 2D USS. It also stated that the 3D USS in comparison to hysteroscopy, the sensitivity and positive predictive value of saline infusion sonography were $98 \%$ and
95\% when performed in combination with 2D USS and $100 \%$ and $92 \%$ with 3D USS respectively.

\section{CONCLUSION}

It is important to investigate the uterine cavity in infertile women with history of frequent, unexplained, and unsuccessful ICSI. 3D USS is known to have a good sensitivity and specifity for diagnosis of uterine cavity abnormalities especially in differentiating between different types of uterine anomalies. It is of great value in delineating the exact position of submucous myoma and intramural myomas that compress and deform uterine cavity. It could be used instead of hysteroscopy as first line of investigation for intrauterine lesions. Hysteroscopy is the gold standard tool and is used as a routine for the assessment of uterine cavity and can be reserved for operative cases or for cases with positive data seen by 3D USS. Hysteroscopy is an invasive procedure and many patients cannot tolerate it, the procedure also has some complications even up to mortality.

\section{REFERENCES}

1. American Society for Reproductive Medicine (2008): Evaluation of the azoospermic male. Fertil Steril., 90(3): S74-S77.

2. Kastrop PM, Weima SM, Van Kooij RT, TeVelde ER (1999): Comparison between intracytoplasmic sperm injection and in vitro fertilization with high insemination concentration after total fertilization failure in a previous IVF attempt. Hum Reprod., 14: 65-69.

3. Palermo GD, Cohen J, Alikani M, Adler A, Rosenwaks Z (1995): Intracytoplasmic sperm injection: a novel treatment for all forms of male factor infertility. Fertil Steril., 63: 12311240.

4. Javed M, Esfandiari N, Casper RF (2010): Failed fertilization after clinical intracytoplasmic sperm injection. Reprod Biomed Online, 20(1):56-67.

5. Arefi S, Soltanghoraee H, Zarnani HA, Tabaei SA, Novin GM, Zeraati H, Ebadi P (2008): Repeated IVF/ICSI-ETs failures and impact of hysteroscopy. Iranian Journal of Reproductive Medicine, 6(1): 19-24.

6. Margalioth EJ, Ben-Chetrit A, Gal M, EldarGeva $T$ (2006): Investigation and treatment of repeated implantation failure following IVF-ET. Hum Reprod., 21(4): 3036-3043. 
7. Stillman RJ, Richter KS, Banks NK, Graha m JR (2009): Elective single-embryo transfer: A 6-year progressive implementation of 784 single blastocyst transfers and the influence of payment method on patient choice. Fertil Steril., 92: 1895-1906.

8. Dunn L, Kelly W, Critchley H (2003): Decidualization of the human endometrial stromal cell: an enigmatic transformation. Reprod Biomed Online, 7: 151-161.

9. Brown SE, Coddington CC, Schnorr J, Toner JP, Gibbons W, Oehninger S (2000): Evaluation of outpatient hysteroscopy, saline infusion hysterosonography and hysterosalpingography in infertile women: A prospective, randomized study. Fertil Steril., 74: 1029-1034.

10. Hinckley MD, Milki AA (2004): 1000 officebased hysteroscopies prior to in vitro fertilization: feasibility and findings. JSLS: Journal of the Society of Laparoendoscopic Surgeons, 8(2): 103-107.

11. Alcázar JL, Aubá M, Olartecoechea B (2012): Three-dimensional ultrasound in gynecological clinical practice, Reports in Medical Imaging. Dovepress J., 5: 1-13.

12. Balen FG, Allen CM, Gardener JE, Siddle NC, Lees WR (1993): Three-Dimensional reconstruction of ultrasound images of the uterine cavity. Br J radiol., 66: 588-591.

13. Bettocchi S, Nappi L, Ceci O, Selvaggi L (2004): Office hystseroscopy. Obstet Gynecol Clin North Am., 31(3): 641-654.

14. Isaacson K (2002): Office hysteroscopy: A valuable but under-utilized technique. Curr Opin Obstet Gynecol., 14: 381-385.

15. Flack VF, Afifi AA, Lachenbruch PA, Schouten HJA (1988): Sample size determinations for the two rater kappa statistic. Psychometrika, 53(3): 321-325.

16. Hintze J (2011): Power Analysis and Sample Size (PASS). https:// www. ncss. com/ support/ faq/

17. Gremeau AS, Andreadis N, Fatum M, Craig J, Turner K, Mcveigh E (2012): In vitro maturation or in vitro fertilization for women with polycystic ovaries? A case-control study of 194 treatment cycles. Fertil Steril., 98(2): 355-360.

18. Simon A, Laufer N (2012): Assessment and treatment of repeated implantation failure (RIF). JAFG: Journal of Assisted Reproduction and Genetics, 29(11): 1227-1239.
19. Jurkovic D (2002): Three-dimensional ultrasound in gynecology: A critical evaluation. Ultrasound Obstet Gynecol., 19: 109-117.

20. George P, Anastasios M (2015): Office Hysteroscopy: Endoscopy-innovative uses and emerging technologies. https:// www. intechopen. $\mathrm{com} / . . . /$ endoscopy-innovative-uses-and-emer.

21. Abdelazim AI, Abo-Elezz A (2012): Complementary roles of hysteroscopy and saline infusion hysterosonography in uterine cavity assessment before in vitro fertilization. Asian Pac J of Reprod., 1(1): 13-16.

22. Khalifa EA, Alsamrah MA, Mahmoud TH, Abdeen NS (2016): Chromohystroscopy after failed intracytoplasmic sperm injection (ICSI). JHIPH: Journal of High Institute of Public Health, 44(1): 48-52.

23. Ebrashy NA, Momtaz M, Shawky AO, Soliman ME, Maaty AZ (2004): Three dimentional transvaginal ultrasound in the assessment of uterine lesions: when do we really need it? Middle East Fertility Society Journal, 9: 79-83.

24. Karasu T, and Metwally M (2017): Preconception risk assessment: gynaecological problems. In Clinical Management of Pregnancies following ART. Springer.

25. Sylvestre C, Child TJ, Tulandi T, Tan SL (2003): A prospective study to evaluate the efficacy of two- and three-dimensional sonohysterography in women with intrauterine lesions. Fertil Steril., 79: 1222-1225. 